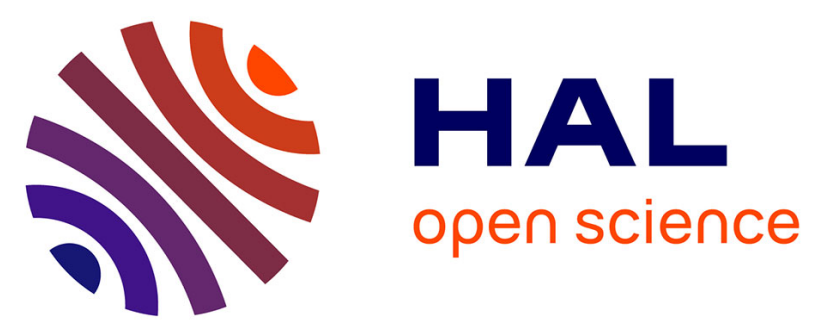

\title{
Improvement in the temporal contrast in the tens of ps range of the multi-PW Apollon laser front-end
}

Lucas Ranc, Catherine Le Blanc, Nathalie Lebas, Luc Martin, Ji-Ping Zou, François Mathieu, Christophe Radier, Sandrine Ricaud, Frédéric Druon, Dimitris Papadopoulos

\section{To cite this version:}

Lucas Ranc, Catherine Le Blanc, Nathalie Lebas, Luc Martin, Ji-Ping Zou, et al.. Improvement in the temporal contrast in the tens of ps range of the multi-PW Apollon laser front-end. Optics Letters, 2020, 45 (16), pp.4599. 10.1364/OL.401272 . hal-02991506

\section{HAL Id: hal-02991506 https://hal.science/hal-02991506}

Submitted on 12 Nov 2020

HAL is a multi-disciplinary open access archive for the deposit and dissemination of scientific research documents, whether they are published or not. The documents may come from teaching and research institutions in France or abroad, or from public or private research centers.
L'archive ouverte pluridisciplinaire HAL, est destinée au dépôt et à la diffusion de documents scientifiques de niveau recherche, publiés ou non, émanant des établissements d'enseignement et de recherche français ou étrangers, des laboratoires publics ou privés. 


\title{
Improvement of the temporal contrast in the tens ps range for the multi-PW Apollon laser front-end
}

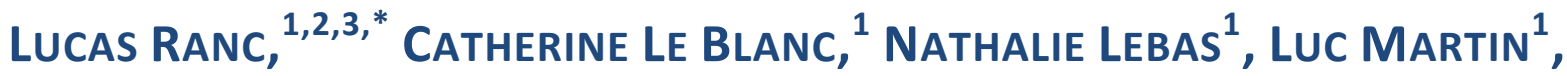 \\ Ji-Ping ZOU ${ }^{1}$, FRANÇOIS MATHIEU ${ }^{1}$, ChRISTOPHE RADIER ${ }^{2}$, SANDRINe RICAUd ${ }^{2}$, \\ FREDERIC DRUON ${ }^{3}$, AND DIMITRIS PAPADOPOULOS ${ }^{1}$ \\ ${ }^{1}$ LULI, CNRS, ECOLE POLYTECHNIQUE Institut Polytechnique de Paris, CEA, SORBONNE UNIVERSITE, 91128 Palaiseau, France \\ ${ }^{2}$ THALES LAS France, Laser Solutions Unit, 2 avenue Gay-Lussac, 78995 Elancourt Cedex, France \\ ${ }^{3}$ Laboratoire Charles Fabry, Institut d'Optique Graduate School, CNRS, Université Paris-Saclay 91127, Palaiseau Cedex, France \\ *Corresponding author: lucas.ranc@polytechnique.edu
}

Received XX Month XXXX; revised XX Month, XXXX; accepted XX Month XXXX; posted XX Month XXXX (Doc. ID XXXXX); published XX Month XXXX

\begin{abstract}
We demonstrate the impact of the optics roughness in Öffner stretchers used in CPA laser chains and how it is possible to improve the temporal contrast ratio in the temporal range of 10-100 ps by adequately choosing the optical quality of the key components. Experimental demonstration has been realized in the frontend source of the multi-PW laser facility "Apollon" resulting in an enhancement of the contrast ratio by 2-3 orders of magnitude. (C) 2019 Optical Society of America
\end{abstract}

http://dx.doi.org/10.1364/OL.99.099999

The continuous evolution of ultra-high-power laser technology gives access to more and more pulse energy and shorter pulse duration reaching peak powers in the range of $10 \mathrm{PW}$ or higher [1]. The ability to focus the laser beam to high intensity allowed recently to reach more than $5 \times 10^{22} \mathrm{~W} / \mathrm{cm}^{2}$ [2]. The temporal contrast ratio (CR) is one of the most important parameters to take into account for laser-matter interaction at such high-intensity levels to avoid any unwanted interaction with the target, significantly corrupting the experiments. Nowadays, emerging PW-laser systems all over the world are continually seeking technical improvements to fulfill the extreme temporal CR requirements. Several methods and strategies have been employed including mostly nonlinear pulse cleaning techniques [3-9] and ultrashort optical parametric chirped pulse amplification (OPCPA) [10]. These techniques are applied principally in the front-end part of the laser systems, in the compressed regime, and are helpful to improve the CR by several orders of magnitude even in the vicinity to the main pulse peak. However, despite all the advances in the CR improvement techniques there is a common temporal feature in all high intensity laser sources, responsible for the degradation of the CR in the 10100 ps range [11-17], as represented schematically in Fig. 1. It appears typically in the front edge of the pulse at about $10^{-5}-10^{-6}$ lower intensity and it follows an approximate exponential decay with a rate of $1-2$ orders of magnitude per $10 \mathrm{ps}$ with a temporal extent of few tens of ps.

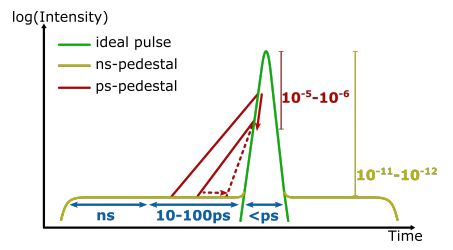

Fig. 1. Typical temporal structure of a laser pulse from a CPA PW system. The red lines represent different ways of improving ps CR by reducing the offset and the temporal extent of the noise pedestal

This specific contrast structure has been the subject of dedicated theoretical and experimental investigations. In Refs. [18,19], complete analytical models of the effect are presented, suggesting two contributions, in the far and near fields, both related to the roughness of optics used in compressors and stretcher in chirped pulse amplification (CPA) systems. In Ref. [20], a different mechanism is suggested based on the nonlinear coupling of postto-pre-pulse long temporal structures. In Ref. [15], Kiriyama et al. discuss the nature of this structure for the case of PW systems, suggesting the contribution of optics in the far field of spectrally dispersed beams. The experimental work in Ref. [21] offers spatially resolved CR measurements supporting the predicted dual contribution [19]. In proof- of-principle experiments, the impact of the Öffner stretcher geometry on the CR for sub-ps Nd:glass lasers has also been shown [22]. Another experimental demonstration in Ref. [13] from the group of Gemini lasers presents interesting results revealing the contribution of the surface quality of the gratings of the compressor and a stretcher in the specific temporal range of the CR. Finally, in recent simulations [23] and in some previous work of ours $[10,24]$, the spatiotemporal coupling as the 
origin for CR degradation has been investigated, pointing out the importance of the Öffner stretchers optics and especially of their primary convex mirror.

The present study describes, to the best of our knowledge, the first experimental proof of the crucial impact of the surface quality of the Öffner stretcher's primary mirror on the contrast of highintensity laser pulses. This work is realized in the Apollon laser facility $[10,11,24]$ and demonstrates a substantial improvement in the CR by two to three orders of magnitude on the $10-100$ ps before the main pulse. More specifically, we both numerically and experimentally explore the spatiotemporal repercussion on the temporal CR, both versus the power spectral density distribution (PSD) of the optical-component surface and the particular spacetime coupling conditions in our setup. All experiments presented hereafter have been realized using a local output of the Apollon laser. The system includes a high-contrast front-end based on an OPCPA operating in the 10 ps range [10] and the first Ti:Sapphire multipass amplifier of the Apollon laser (Fig. 2).

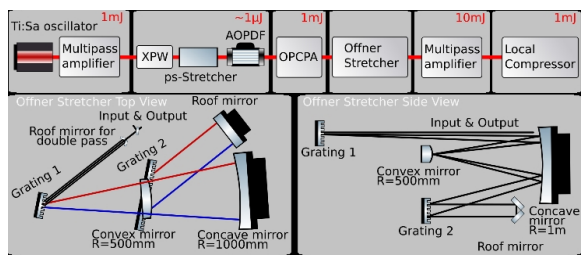

Fig. 2. Typical temporal structure of a laser pulse from a CPA PW system. The red lines represent different ways of improving ps CR by reducing the offset and the temporal extent of the noise pedestal

The specific amplifier, operating at $10 \mathrm{~Hz}$, allows the parametric study of the CR at the suitable energy level, based on a standard third order cross-correlation scanning device. Additionally, as the highest small signal gain amplification stage in the Apollon system $(\sim \times 3000)$ is representative of potential amplified spontaneous emission (ASE) degradation. The pulses are then compressed in a folded two gratings compressor (using $1480 \mathrm{l} / \mathrm{mm}$ gold gratings), providing $\sim 1 \mathrm{~mJ}$ at $20 \mathrm{fs}$, close to the Fourier transform limit (17 fs) with beam size of $\sim 1 \mathrm{~cm}\left(1 / \mathrm{e}^{2}\right)$. The Apollon Öffner stretcher (lower part of Fig. 2) is based on the double grating configuration $[25,26]$. The first grating is precisely placed at the center of the curvature of the concave and the convex mirrors in order to reduce the spatiotemporal aberrations. Precise alignment, based on the combination of standard techniques and the use of a specific 3-color narrow linewidth laser diode system [26] guarantees the required precision. Roof mirrors are used to double pass the stretcher. As explained in Refs [18-19] we can anticipate two distinct CR degradation phenomena due to the scattering of optics where the beam is spectrally dispersed, in stretcher and compressor systems. The first one is a pure axial contribution generated by optics located in the Fourier plane: the only component in our case is the convex mirror of the Öffner-stretcher. The second one is a non-axial contribution, generated by optics in the near-field plane - e.g. gratings, concave mirror or roof mirrors in folded configurations. Furthermore, in this case the degradation of the contrast is heavily impaired by the often much larger beam size (of each individual spectral component of the pulse) smoothing out the high frequency terms and limiting the temporal range of their impact close to the main pulse peak. This study gives an emphasis on the axial contributions since these are responsible for the effective CR observed on the target after focusing [19]. Our experimental investigation is organized in two steps. We first consider the impact of the convex mirror surface quality. As detailed in Refs. [18], the PSD of the mirror is imprinted on the contrast curve as shown in formula 10 of this article. The low spectral frequencies of the mirror PSD impact the short time range of the CR and the highest ones impact the longest time scales. Quantitatively, the CR is proportional to the square of the RMS value of the mirror roughness $\sigma^{2}$ and therefore experimentally we expect an improvement of the CR following the solid arrow indication in Fig. 1. Second, we study the modification of the spatiotemporal coupling conditions by adjusting the beam size on the convex mirror. In fact, increasing its size on the convex mirror (by reducing the beam size at the input of the stretcher) accentuates the smoothing effect of the higher spatial frequency surface defects and consequently the fast modulating spectral phase terms. We therefore, this time expect a restriction of the noise pedestal temporal extend closer to the main pulse peak, as indicated by the dashed arrow in Fig. 1. We compare two convex mirrors, a standard high-quality one, $\mathrm{CX}_{1}$ and a custom one, $\mathrm{CX}_{2}$, manufactured under the tightest possible commercially available specifications regarding both the global RMS value of the roughness and the restriction of the bandwidth of its PSD.

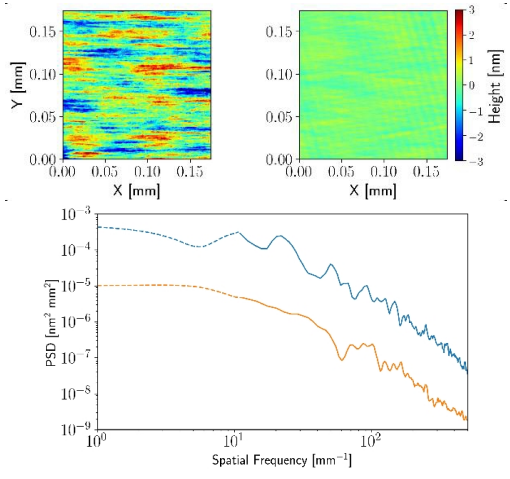

Fig. 3. (top) Measured roughness of a standard convex mirror (CX1) versus the custom one (CX2) of $1.02 \mathrm{~nm}$ (left) and $0.17 \mathrm{~nm}$ (right) RMS, respectively. (down) Estimated 2D-PSD of a standard (blue) convex mirror versus the custom one (orange). The dashed-line part of the curves corresponds to a mathematical extension of the PSD according to the specifications of the mirrors

The roughness measurements of these mirrors, shown in Fig. 3, have been performed with optical surface profilometers (OptoSURF from Eotech for the $\mathrm{CX}_{1}$ and a ZYGO NewView 9000 for $\mathrm{CX}_{2}$ ). Both measurements are based on phase shifting microinterferometry with similar depth resolution $(<0.1 \mathrm{~nm})$ and magnification (x50). The measured RMS values for the roughness of the two mirrors are $1.02 \mathrm{~nm}$ and $0.17 \mathrm{~nm}$ for the $\mathrm{CX}_{1}$ and $\mathrm{CX}_{2}$ respectively. It should be noted that the measurement of $\mathrm{CX}_{1}$ is done after coating while for $\mathrm{CX}_{2}$ it was only possible for the uncoated substrate during the fabrication process. The coating process however of $\mathrm{CX}_{2}$ has been qualified by the supplier on flat optics to have a marginal impact on the roughness of the final component. In Fig. 3, we provide the 2D-PSD functions of the two convex mirrors. For our simulation, we use a 2D-to-1D-PSD 
transformation based on an azimuthal averaging function [27] of the measured surface, given by the formula (4) in Ref. [27].

The measurement of the PSD has been realized over several small fractions of the mirror of $200 \times 200 \mu \mathrm{m}^{2}$. This restricts the information of the spatial frequencies of the mirror defects in the $10-10^{3} \mathrm{~mm}^{-1}$ range and the corresponding spectral phase in a range that limits the temporal contrast representation between about $10-1000$ ps. While this temporal range is enough for the purpose of this study, in order to obtain a more realistic estimation of the contrast, we have mathematically completed the measured PSD (dashed-line curve parts of the Fig. 3 (down) graphs) following the specification of the mirrors down to $1 \mathrm{~mm}^{-1}$. Then, this completed PSD has been used as a filter of a Gaussian random noise signal in order to create the surface of the complete convex mirror. As the convex mirror is located in a Fourier plane, in our 1D simulation model, we directly consider that each frequency component of the pulses experiences a phase shift $\Delta \varphi(\omega)=N(2 \Delta \mathrm{h} \omega / \mathrm{c})$ assuming a linear repartition of $\omega$ over the length of the mirror and with $\mathrm{N}$ the total number of reflections of the beam on the mirror $(\mathrm{N}=4$ in our case). The impact of the beam size at the Fourier plane is taken into account by applying a sliding average on the function $\Delta \varphi(\omega)$ over $\omega$. Our 1-D model is voluntarily simplified to be usable with accessible standard data and does not take into account all the potential effects, described in more detail elsewhere [23] Its purpose is to provide a clear insight on the basic mechanism and the expected tendencies on the CR modification.

In Fig. 4 (top, inset), we show the corresponding spectral phase profiles (presented here only over a restricted spectral range of 2 $\mathrm{nm}$ for clarity reasons) of the $\mathrm{CX}_{2}$ mirror for a beam size of $6.4 \mathrm{~mm}$ (orange) and $3.2 \mathrm{~mm}$ (green) at the input of the stretcher as well as for no sliding average (black). In Fig. 4 (top) we present the simulation of the CR based on the PSD function of the $\mathrm{CX}_{1}$ (blue curve) and the $\mathrm{CX}_{2}$ (orange and green curve) with respectively beam sizes at the input of the stretcher of $6.4 \mathrm{~mm}, 6.4 \mathrm{~mm}$ and 3.2 $\mathrm{mm}$ (or $80 \mu \mathrm{m}$ and $160 \mu \mathrm{m}$ respectively on the convex mirror). Our simulation clearly predicts a positive impact of the increased quality of the $\mathrm{CX}_{2}$ : the noise pedestal is reduced by 2-3 orders of magnitude. Additionally, spatial averaging also reduces the temporal extent from $\sim 50$ ps to $\sim 25 \mathrm{ps}$.

For our experimental demonstration, the CR measurements have been realized with a high-dynamic range cross-correlator (Sequoia, from Amplitude Technologies). Careful alignment of the device allowed a dynamic range between $10^{11}$ and $10^{12}$. Given the ASE level of the source [10], estimated in the $10^{-13}$ range, and using multiple repeated measurements, we can fairly attribute the background level of all the CR measurements in this work to the detector's noise level. The first set of measurements has been realized with the $\mathrm{CX}_{1}$ mirror. As a variable parameter for these measurements, we adjusted the repartition of the gain between the OPCPA and the Ti:Sa amplifier in order to reach a constant output energy. For a gain variation in the OPCPA between 1001000 , no measurable difference could be observed on the CR. In fact, as explained in Refs. [10, 24], the noise contribution of the OPCPA, due to its low gain and the temporal extend of the OPCPA pump $(<15 \mathrm{ps})$, is negligible compared to other contributions in the same temporal range. A typical CR curve of this first set of measurements is shown in Fig. 4 (down, blue line curve). We observe that the noise pedestal has an almost perfect exponential shape with a starting point at around $10^{-5}$ just before the main peak and a decay rate of about 1 order of magnitude every 10 ps.
The dynamic range of this measurement is $10^{11}$ allowing an accurate measurement of the noise structure over more than $50 \mathrm{ps}$ before the pulse.
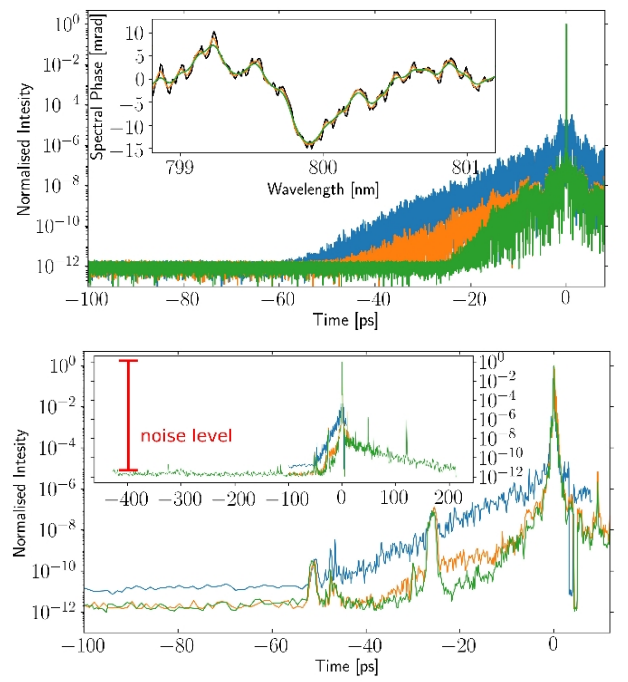

Fig. 4. (top) $1 \mathrm{D}$ simulation of the temporal CR for the case of $\mathrm{CX}_{1}$ (blue) and $\mathrm{CX}_{2}$ (orange and green) with a beam size of $6.4 \mathrm{~mm}, 6.4 \mathrm{~mm}$ and $3.2 \mathrm{~mm}$ respectively. Inset: Spectral phase modulation related to $\mathrm{CX}_{2}$ (black) and $\mathrm{CX}_{2}$ sliding average for a beam size of $6.4 \mathrm{~mm}$ (orange) and $3.2 \mathrm{~mm}$ (green), represented on $2 \mathrm{~nm}$ for clarity. The overall sliding average only marginally affects the low frequencies and the phase rms value remains practically unchanged. (down) Temporal CR measurement comparison between the scattering of CX1 (blue) and CX2 (orange), and the averaging effect (green). The three different sets of measurements show different background levels of about 10-11 and $2 \times 10-12$ as result of finely different

The second set of measurements has been realized with the $\mathrm{CX}_{2}$, replacing $\mathrm{CX}_{1}$ in the stretcher. This operation has been carefully realized so that the new mirror is installed in the exact same position of the $\mathrm{CX}_{1}$. A precise alignment is performed using near and far field references at the output of the stretcher together with injecting a 3-color laser reference beam. The angular and centering precision in this replacement procedure is estimated to be better than $2 \mu \mathrm{rad}$ and $50 \mu \mathrm{m}$ respectively. Furthermore, the pulse duration has been monitored during the process with nonmeasurable differences between the two mirrors ( $\sim 20 \pm 1 \mathrm{fs})$.

The measured $\mathrm{CR}$ with the $\mathrm{CX}_{2}$ is show in Fig. 4 (down, orange line curve). A reduction of the noise level by 2-3 orders of magnitude between the $\mathrm{CX}_{1}$ (blue line) and $\mathrm{CX}_{2}$ is directly observed. At -13 ps for example the pedestal level difference decreases from $3 \times 10^{-7}$ down to $3 \times 10^{-10}$. Close to the main peak, and in the temporal range impacted by the lower spatial frequency components of the mirror PSD, the improvement is less impressive, $\sim \times 10-50$, which is in agreement with the ratio of the squared RMS values of the roughness of the two mirrors $(\sim 40)$. The temporal extend of the pedestal is reduced as well, reaching the measurement noise level $\left(<5 \times 10^{-12}\right)$ at about -37 ps. Compare to $\mathrm{CX}_{1}$ this corresponds to a temporal extent restriction of the pedestal of about $20 \mathrm{ps}$.

The last set of measurements has been finally realized after the modification of the beam size on the $\mathrm{CX}_{2}$. This has been done by 
simply temporarily suppressing a lens-based $\times 2$ beam-expander at the input of the stretcher, increasing therefore the beam size of each spectral component on the convex mirror by the same factor (from $80 \mu \mathrm{m}$ to $160 \mu \mathrm{m}$ on the convex mirror). We clearly observe in the green line curve of Fig. 4 (down) the suppression of the noise structure in the -13 ps to -37 ps range. This is in agreement with the predicted behavior of the CR in Fig. 4 (top) in the case of $\times 2$ larger beam on the $\mathrm{CX}_{2}$.

For the sake of completeness, the variation of the OPCPA gain has been done again for both beam size cases with the $\mathrm{CX}_{2}$ mirror resulting once more in no measurable variation of the $\mathrm{CR}$. Despite the relatively good agreement with our numerical prediction, it should be noted that, in this last configuration, other potential effects might also play a role. Among them, the increased divergence of the smaller input beam and residual collimation errors could lead to non-negligible longitudinal mismatch of the beam focal plane and the $\mathrm{CX}_{2}$ plane which could then alter the exact space-spectral coupling condition and the expression of the $\mathrm{CR}$ in the far field of the beam. The observed temporal asymmetry in the green line curve (inset of Fig. 4 down) can certainly not be attributed to any phase effect and therefore to the CX mirror surface quality. It reveals the impact of a different mechanism, responsible for the degradation of the contrast in the falling side of the contrast curve, which is however out of the scope of this study. A side effect of the reduction of the exponential noise pedestal by the improvement of the CX mirror quality has been the revelation of few pre-pulses, already apparent in the initial curve, but only slightly above the noise. These peaks have clearly no reason to be impacted by the CX mirror roughness and keep the same amplitude. These peaks (in particular the ones at -52 and -26 ps) constitute a very practical, robust, and straightforward calibration of the CR curves, validating then quantitatively the $\mathrm{CR}$ ratio improvement. We can probably associate the three events at -52 ps, -26 ps and +26 ps and assign the pre-pulses to nonlinear coupling mechanisms of post-to-pre structures [28]. The origin of the multiple-peak group of pre-pulses at -49 ps, on the other hand is undoubtedly a double reflection of the 4-mm-thick $\beta-\mathrm{BaB}_{2} \mathrm{O}_{4}$ (BBO) crystal of the OPCPA stage. We could then reduce its impact by deliberately tilting the $\mathrm{BBO}$ crystal $\left(\sim 5^{\circ}\right)$.

In conclusion, we demonstrate, to the best of our knowledge, the first experimental demonstration of the impact of the surface quality of the convex mirror in an Öffner stretcher on the CR of high intensity pulses. Replacement of this component resulted in an impressive improvement of the CR in the range of 10-100 ps of the Apollon laser source. Furthermore, we suggest an engineering approach for the restriction of the temporal extend of the specific noise pedestal based on the modification of the beam size at the input of the stretcher.

Funding. ILE APOLLON (07-CPER 017-01); Labex PALM (ANR10-LABX-0039-PALM, 2impacts)

Acknowledgment. The authors gratefully thank Muriel Thomasset (Synchrotron SOLEIL) for her contribution on the surface roughness measurements of $\mathrm{CX}_{1}$.

Disclosures. The authors declare no conflicts of interest.

\section{REFERENCES}

1. C. N. Danson, C. Haefner, J. Bromage, T. Butcher, J.-C. F. Chanteloup, E. A. Chowdhury, A. Galvanauskas, L. A. Gizzi, J. Hein, D. I. Hillier, N. W. Hopps, Y. Kato, E. A. Khazanov, R. Kodama, G. Korn, R. Li, Y. Li, J. Limpert, J. Ma, C. H. Nam, D. Neely, D. Papadopoulos, R. R. Penman, L. Qian, J. J. Rocca, A. A. Shaykin, C. W. Siders, C. Spindloe, S. Szatmári, R. M. G. M. Trines, J. Zhu, P. Zhu, and J. D. Zuegel, High Power Laser Sci. Eng. 7, e54 (2019).

2. J. H. Sung, H. W. Lee, J. Y. Yoo, J. W. Yoon, C. W. Lee, J. M. Yang, Y. J. Son, Y. H. Jang, S. K. Lee, and C. H. Nam, Opt. Lett. 42, 2058 (2017).

3. L. P. Ramirez, D. Papadopoulos, M. Hanna, A. Pellegrina, F. Friebel, P. Georges, and F. Druon, J. Opt. Soc. Am. B 30, 2607 (2013).

4. D. Homoelle, A. L. Gaeta, V. Yanovsky, and G. Mourou, Opt. Lett. 27, 1646 (2002).

5. N. Minkovski, G. I. Petrov, S. M. Saltiel, O. Albert, and J. Etchepare, J. Opt. Soc. Am. B 21, 1659 (2004).

6. G. Doumy, F. Quéré, O. Gobert, M. Perdrix, P. Martin, P. Audebert, J. C. Gauthier, J.-P. Geindre, and T. Wittmann, Phys. Rev. E 69, 026402 (2004).

7. A. Jullien, O. Albert, F. Burgy, G. Hamoniaux, J.-P. Rousseau, J.-P. Chambaret, F. Augé-Rochereau, G. Chériaux, J. Etchepare, N. Minkovski, and S. M. Saltiel, Opt. Lett. 30, 920 (2005).

8. J. Liu, K. Okamura, Y. Kida, and T. Kobayashi, Opt. Express 18, 22245 (2010).

9. X. Shen, P. Wang, J. Liu, and R. Li, High Power Laser Sci. Eng. 6, e23 (2018).

10. D. N. Papadopoulos, P. Ramirez, K. Genevrier, L. Ranc, N. Lebas, A. Pellegrina, C. Le Blanc, P. Monot, L. Martin, J. P. Zou, F. Mathieu, P. Audebert, P. Georges, and F. Druon, Opt. Lett. 42, 3530 (2017).

11. D. Papadopoulos, J. Zou, C. Le Blanc, G. Chériaux, P. Georges, F. Druon, G. Mennerat, P. Ramirez, L. Martin, A. Fréneaux, A. Beluze, N. Lebas, P. Monot, F. Mathieu, and P. Audebert, High Power Laser Sci. Eng. 4, e34 (2016).

12. Z. Li, S. Tokita, S. Matsuo, K. Sueda, T. Kurita, T. Kawasima, and N. Miyanaga, Opt. Express 25, 21201 (2017).

13. C. Hooker, Y. Tang, O. Chekhlov, J. Collier, E. Divall, K. Ertel, S. Hawkes, B. Parry, and P. P. Rajeev, Opt. Express 19, 2193 (2011).

14. L. Yu, Y. Xu, Y. Liu, Y. Li, S. Li, Z. Liu, W. Li, F. Wu, X. Yang, Y. Yang, C. Wang, X. Lu, Y. Leng, R. Li, and Z. Xu, Opt. Express 26, 2625 (2018).

15. H. Kiriyama, A. S. Pirozhkov, M. Nishiuchi, Y. Fukuda, K. Ogura, A. Sagisaka, Y. Miyasaka, M. Mori, H. Sakaki, N. P. Dover, K. Kondo, J. K. Koga, T. Z. Esirkepov, M. Kando, and K. Kondo, Opt. Lett. 43, 2595 (2018).

16. Y. Wang, S. Wang, A. Rockwood, B. M. Luther, R. Hollinger, A. Curtis, C. Calvi, C. S. Menoni, and J. J. Rocca, Opt. Lett. 42, 3828 (2017).

17. K. Nakamura, H.-S. Mao, A. J. Gonsalves, H. Vincenti, D. E. Mittelberger, J. Daniels, A. Magana, C. Toth, and W. P. Leemans, IEEE J. Quantum Electron. 53, 1 (2017).

18. C. Dorrer and J. Bromage, Opt. Express 16, 3058 (2008).

19. J. Bromage, C. Dorrer, and R. K. Jungquist, J. Opt. Soc. Am. B 29, 1125 (2012).

20. N. Khodakovskiy, M. Kalashnikov, E. Gontier, F. Falcoz, and P.-M. Paul, Opt. Lett. 41, 4441 (2016).

21. J. Ma, P. Yuan, J. Wang, Y. Wang, G. Xie, H. Zhu, and L. Qian, Nat. Commun. 6, 6192 (2015).

22. X. Lu, X. Wang, Y. Leng, X. Guo, Y. Peng, Y. Li, Y. Xu, R. Xu, and X. Qi, IEEE J. Sel. Top. Quantum Electron. 24, 1 (2018).

23. V. A. Schanz, M. Roth, and V. Bagnoud, J. Opt. Soc. Am. A 36, 1735 (2019).

24. L. Ranc, J.-P. Zou, C. Le Blanc, C. Radier, C. Simon-Boisson, F. Druon, and D. N. Papadopoulos, in International Committee on Ultrahigh Intensity Lasers (ICUIL), Lindau, Germany, 2018.

25. G. Cheriaux, B. Walker, L. F. Dimauro, P. Rousseau, F. Salin, and J. P. Chambaret, Opt. Lett. 21, 414 (1996).

26. C. Le Blanc, I. Taghzout, J.-P. Zou, F. Druon, and D. Papadopoulos, 373 in International Committee on Ultrahigh Intensity Lasers (ICUIL), 374 Montebello,Western Quebec, Canada, 2016

27. E. Sidick, Proc. SPIE 7390, 73900L (2009).

28. N. V. Didenko, A. V. Konyashchenko, A. P. Lutsenko, and S. Y. Tenyakov, Opt. Express 16, 3178 (2008). 